\title{
An experimental study of the active control of multiple-wave types in an elastic beam
}

\author{
R. L. Clark, Jr. \\ Department of Mechanical Engineering, Virginia Polytechnic Institute and State University, Blacksburg, \\ Virginia 24061 \\ Jie Pan and Colin H. Hansen \\ Department of Mechanical Engineering, University of Adelaide, South Australia, 5001, Australia
}

(Received 22 August 1991; revised 2 January 1992; accepted 29 January 1992)

\begin{abstract}
In many practical situations, different types of waves may coexist within a structure. If control is limited to a single wave type, other kinds of waves may be generated unintentionally by the action of the control forces. In this paper an experimental study of the simultaneous control of multiple wave types in a semi-infinite beam has been undertaken. The ability of the piezoelectric control sources to provide adequate system control and the ability of the accelerometer error sensors to provide adequate observability of the power flow associated with each wave type was also evaluated.
\end{abstract}

PACS numbers: 43.40.Cw, 43.40.Vn

\section{INTRODUCTION}

Active control of beam vibration has been the subject of much past research because the characteristics of the beam are well understood and the beam itself is a fundamental element of many complex structures. Vibrations in the beam, traveling in the form of flexural, extensional, or torsional waves, can transfer energy to other components of a structure, resulting in undesired structure response or sound radiation. Much of the past work has been devoted to controlling flexural waves in thin beams since this wave type corresponds to the largest transverse displacement. ${ }^{1-5}$ While flexural waves certainly contribute most significantly to sound radiation from the beam itself, extensional waves cannot be ignored, especially when the beam is a component of a larger superstructure. When extensional waves contact a discontinuity, such as a member connected perpendicularly to the beam, large out-of-plane motions can be excited, resulting in the conversion of extensional wave energy to flexural wave energy. This need to control multiple wave types in beams has been demonstrated by Pan and Hansen. ${ }^{6,7}$

Fuller et al. were the first group to address active control of both flexural and extensional power flow experimentally. However, the work was limited to a thin beam, supporting only one flexural wave. ${ }^{8}$ In this particular study, piezoceramic elements were bonded symmetrically to the surface of the beam to provide control inputs to the structure. If the two elements are wired such that extension is induced on one surface while contraction is induced on the other upon application of a cyclic voltage, uniform bending about the neutral axis results, and flexural waves can be controlled or generated. However, if the symmetric elements are wired such that both are in extension (or contraction) simultaneously, only extensional waves are controlled or generated. In the previous work, ${ }^{8}$ the elements were wired independently and an adaptive LMS algorithm was used to determine the optimal voltage and phase between each piezoelectric element to simultaneously control extensional and flexural waves when necessary.

Actuators used in the work reported here consisted of two piezoelectric elements mounted on opposite faces of the beam and wired electrically to control either flexural or extensional waves. As a thick beam was used, two flexural waves could be driven simultaneously. Thus, two pairs of elements were driven with the required relative phase relationship to control two flexural waves and one pair of elements was driven with the required phase relationship to control the extensional wave. Each actuator pair thus contributed to a single wave type.

To achieve control, the multichannel version of the filtered-x adaptive LMS algorithm was implemented on three control channels to compute the optimal voltages required to control two flexural waves and one extensional wave simultaneously. The error signals were obtained from accelerometers located on the beam and combined using an analog computer such that only one extensional or flexural wave to be controlled was observed by each error input to the controller. Upon achieving control, the displacement and rotational response of the beam was measured at cross sections along the length of the beam to evaluate the active control effectiveness.

\section{ACTIVE CONTROL SYSTEM}

An active control system can be divided generally into two subsystems. One is the physical system, consisting of the system to be controlled (such as the vibration field in a structure) as well as the control actuators and the error sensors. The other subsystem is the "electronic control" system, which takes the information from the error sensor and drives the control actuators according to a cost function implemented in the control algorithm. Figure 1 shows a schematic 
PZT

ACC.

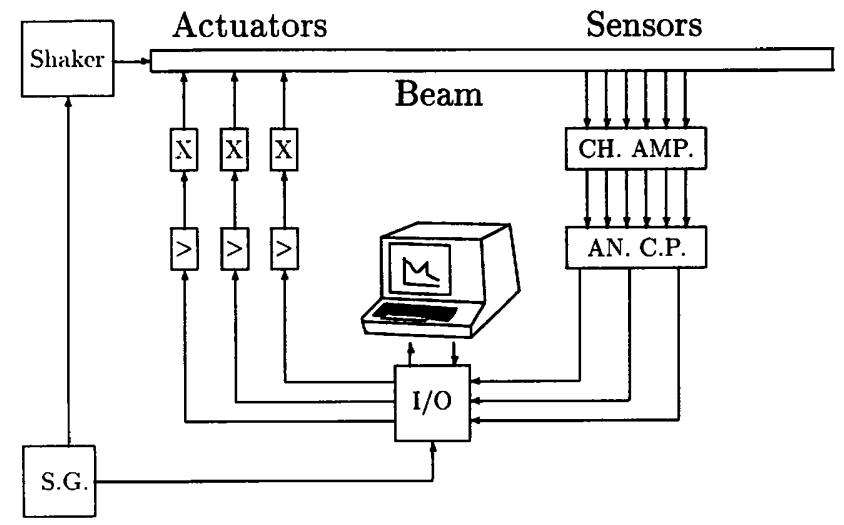

FIG. 1. Schematic diagram of experimental setup for controlling multiple wave types in a beam. $X=$ transformer, $>=$ amplifier, CH.AMP. $=$ charge amplifier, AN. C.P. $=$ analog computer, S.G. = signal generator.

diagram of the experimental arrangement for controlling multiple wave types in a beam. The physical system consists of an aluminum beam of cross sectional dimensions $25 \times 50$ $\mathrm{mm}$ and a length of $4 \mathrm{~m}$. One end $(x=0)$ is driven by a mechanical shaker and the other end is located in a sand box to provide a semi-anechoic termination. The shaker is oriented off-axis at an angle such that all wave types in the beam can be excited. Figure 2(a) and (b) shows the beam, the primary mechanical shaker, and the coordinate system. The possible wave types propagating along the beam are flexural

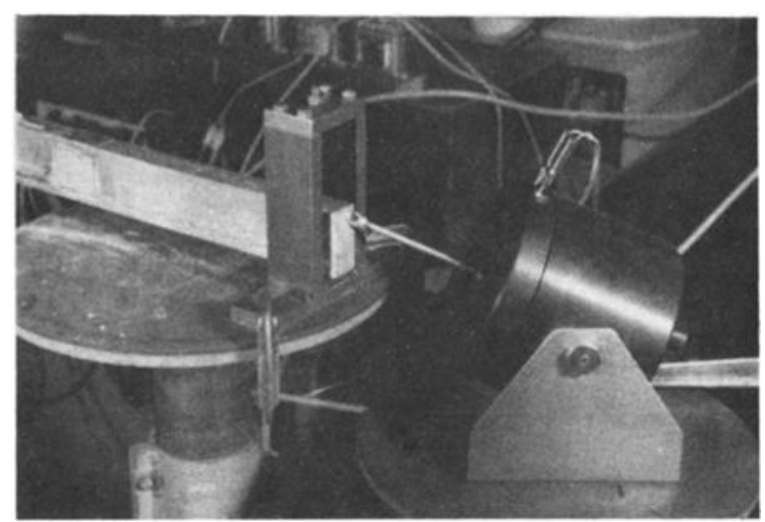

(a)

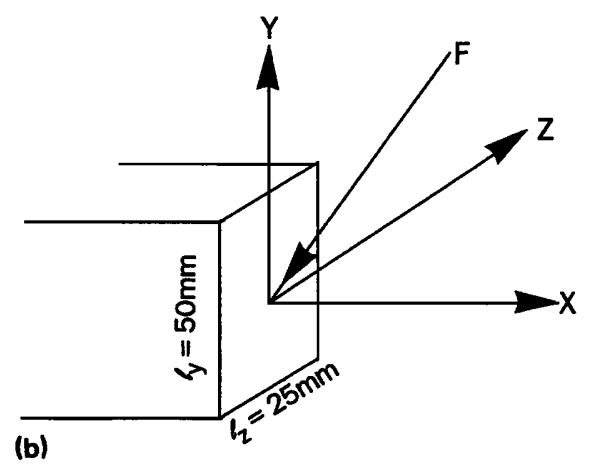

FIG. 2. Aluminum beam with the primary mechanical shaker at one end of the beam: (a) shaker attachment; (b) coordinate system. flexural wave excitation

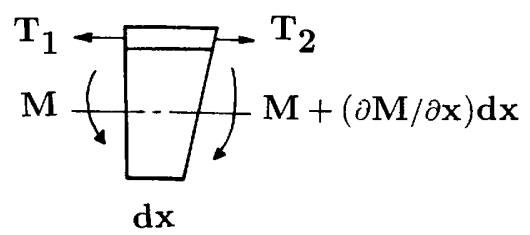

longitudinal wave excitation

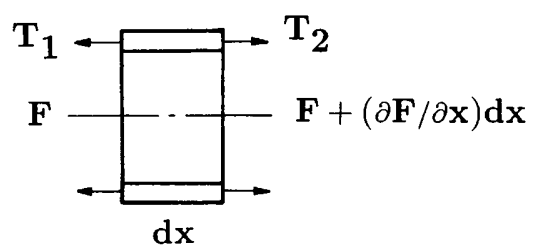

FIG. 3. Piezoelectric actuator configurations: (a) actuators for controlling flexural wave types; (b) actuators for controlling longitudinal wave types.

waves (vibrating in two orthogonal beam planes), longitudinal waves, and torsional waves. Because control was limited to three channels for the experiment (due to hardware limitations), only three outputs to the control actuators and three error signals from the accelerometer-based error sensors were utilized.

\section{A. Actuator arrangement}

The control actuators were constructed using thin piezoelectric elements (lead zirconate titanate). When a cyclic voltage is applied across the thickness of a piezoelectric element bonded to the surface of the beam, the element expands and contracts in its plane. Due to the strain continuity on the bonding structure, the in-plane stress induced by the voltage applies a bending moment to the attached beam [see Fig. 3 (a) ]. A control actuator will be defined here as two piezoelectric elements bonded symmetrically to either side of the beam and wired such that the applied voltage elicits uniform bending about the neutral axis or uniform extension along the axis as outlined by Crawley and de Luis. ${ }^{9}$

Since two flexural waves can exist simultaneously in the beam, two sets of actuators were configured to control each

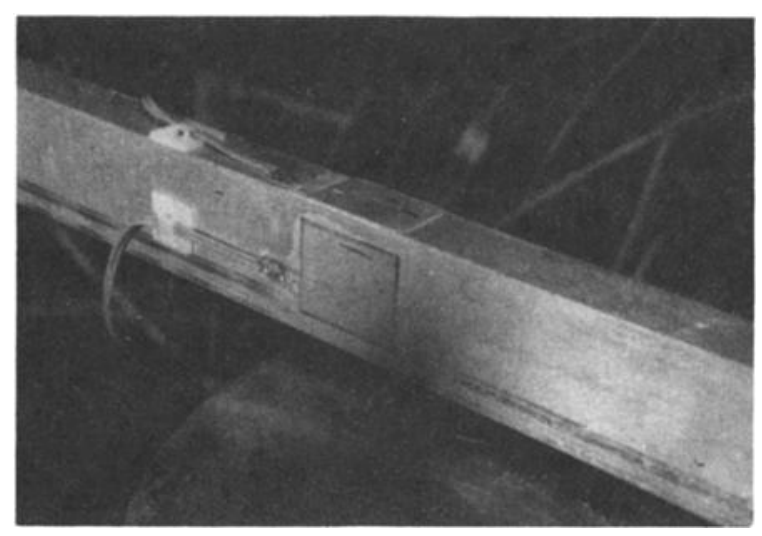

FIG. 4. Photo of the piezoelectric actuators on the beam surfaces. 
wave type. According to its propagating and vibrating directions, one flexural wave is denoted as the $(X-Y)$ flexural wave and the other is denoted as the $(X-Z)$ flexural wave. The center of the piezoelectric actuator for controlling the $(X-Y)$ flexural wave is located at $x=0.25 \mathrm{~m}$, with one element of the actuator bonded to the $\left(x, y=l_{y} / 2\right)$ and one bonded to the $\left(x, y=-l_{y} / 2\right)$ surface. The center of the actuator for controlling the $(X-Z)$ flexural wave is also located at $x=0.25 \mathrm{~m}$, with one element of the actuator bonded to the $\left(x, z=l_{z} / 2\right)$ surface and one bonded to the $(x, z$ $=-l_{z} / 2$ ) surface. Figure 4 shows the two flexural wave actuators on the beam. The center of the piezoelectric actuator required to control the longitudinal waves is located at $x=1.0 \mathrm{~m}$. One element is bonded to the $\left(x, z=l_{z} / 2\right.$ surface and the other to the $\left(x, z=-l_{z} / 2\right)$ beam surface.

The three control actuators were driven by the electrical signals generated with the active controller. As shown in Fig. 1, the control signals were amplified using three separate power amplifiers, and the driving voltage was further increased with transformers having a step-up ratio of 20:1.

\section{B. Sensor arrangement}

The error sensors were developed by using six accelerometers (located at the $x=2.03-\mathrm{m}$ beam cross section) and an analog computer. As shown in Fig. 5(a), two accelerometers located on the $\left(x, y=l_{y} / 2\right)$ and $\left(x, y=-l_{y} / 2\right)$ beam surfaces, respectively, were used to sense the $(X-Y)$ flexural wave. The outputs from these two accelerometers were pre-amplified and subtracted in an analog computer to obtain a voltage that is proportional to the local acceleration of the flexural wave component. Similarly, the outputs from the other two accelerometers located on $\left(x, y=l_{z} / 2\right)$ and $\left(x, y=-l_{z} / 2\right)$ were subtracted to yield the other flexural

flexural wave measurement

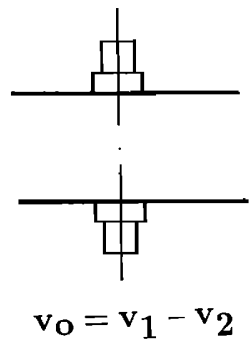

longitudinal wave measurement

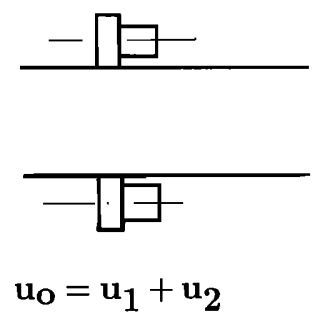

FIG. 5. Accelerometer sensor configurations: (a) accelerometers of sensing flexural wave types; (b) accelerometers for sensing longitudinal wave types. wave component. Finally, to measure the longitudinal wave component in the $X$ direction, two accelerometers were located on the side of the beam [on the $\left(x, y=l_{y} / 2\right)$ and $(x, y$ $=-l_{y} / 2$ ) beam surfaces respectively], pointing in the $X$ direction as shown in Fig. 5(b). The outputs from these were summed with the analog computer to obtain a voltage proportional to the local acceleration of the longitudinal wave component. The three outputs from the analog computer were fed into the controller as the error signals to be minimized.

\section{Description of controller}

To achieve control, a three-channel adaptive controller based on the multi-channel version of the filtered- $x$ LMS control algorithm was implemented on a TMS320C25 DSP resident in an AT computer. This algorithm has been described in detail by Elliot et al. ${ }^{10,11}$ A block diagram illustrating the controller is presented in Fig. 6. The output of the $l$ th error sensor can be modeled at the $n$th time step as

$e_{l}(n)=d_{l}(n)+\sum_{m=1}^{3} \sum_{j=0}^{N-1} P_{l m j} \sum_{i=0}^{N-1} w_{m i}(n-j) x(n-i-j)$,

where $d_{l}(n)$ is the response to the primary excitation at the $l$ th error sensor, $x(n)$ is the input reference signal, $w_{m i}$ are the coefficients of the adaptive FIR filters, and $P_{l m j}$ is the $j$ th coefficient of the transfer function between the output of the $m$ th adaptive filter and the output of the $l$ th error sensor. The number of filter coefficients is denoted by $N$.

To develop the LMS algorithm, the squared error signal is defined by

$$
J=E\left(\sum_{l=1}^{3} e_{l}^{2}(n)\right),
$$

where $E$ is the expectation operator. Since $J$ is a quadratic function of the filter weight coefficients $w_{m i}$, only one minimum solution for $J$ exists.

The outputs of the fixed filters, $P_{l m j}^{\prime}$ (which are estimates of the actual coefficients $\boldsymbol{P}_{l m j}$ describing the actual transfer functions), at each time step $n$, were used by the LMS algorithm to minimize the squared error signal by updating the weight coefficients of the adaptive filter as follows:

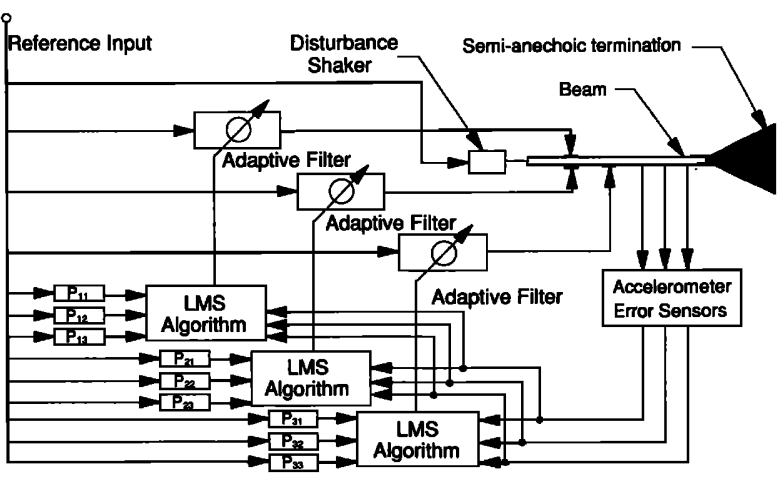

FIG. 6. Controller block diagram. 


$$
w_{m i}(n+1)=w_{m i}(n)-\mu \sum_{l=1}^{3} e_{l}(n) r_{l m}(n-i),
$$

where

$$
r_{l m}(n-i)=\sum_{j=0}^{N-1} P_{l m j}^{\prime} x(n-i-j) .
$$

Note that the summation in Eq. (3) is an estimate of the gradient necessary for updating the weight coefficients. The filtered signals $r_{l m}(n)$, are the outputs of the compensating filters characterized by coeficients $\boldsymbol{P}_{l m j}^{\prime}$ that are measured prior to starting the control algorithm. The factor $\mu$ in Eq. (3) is the algorithm convergence coefficient which regulates the controller speed and stability during convergence.

In the experiment, a signal generator resident on the Bruel and Kjaer model 2034 spectrum analyzer was used to create a steady-state sinusoidal disturbance and reference signal for the LMS controller. Since the signal was sinusoidal, a finite impulse response filter with two coeficients was implemented in the controller transversal filter shown in Fig. 6. The choice of sampling rate was based upon the frequency of the disturbance to optimize the controller performance. For an excitation frequency of $970 \mathrm{~Hz}$, the sampling rate was $9.7 \mathrm{kHz}$ (that is, approximately 10 times the frequency to be controlled).

\section{BEAM STRUCTURAL RESPONSE}

The vibration distribution in the beam for each wave type was compared for both controlled and uncontrolled conditions. At each beam cross section, six global displacement components are required to describe the vibration field in the cross section. Three of them $\left[\bar{u}_{0}, \bar{v}_{0}, \bar{w}_{0}\right]$ describe the translations of the cross section and the other three $\left[\bar{\theta}_{x}, \bar{\theta}_{y}, \bar{\theta}_{z}\right]$ describe the rotations of the cross section. These six acceleration components can be determined from the acceleration component measurements on the surface of the cross section. ${ }^{12}$ Figure 7 shows the arrangement of nine accelerometers at three positions on a beam cross section. The local acceleration components (three orthogonal components at one point) measured at these three locations$\omega^{2}\left[\bar{u}_{1}, \bar{v}_{1}, \bar{w}_{1}, \bar{u}_{2}, \bar{v}_{2}, \bar{w}_{2}, \bar{u}_{3}, \bar{v}_{3}, \bar{w}_{3}\right]$-are related to the global displacement components $\left[\bar{u}_{0}, \bar{v}_{0}, \bar{w}_{0}, \bar{\theta}_{x}, \bar{\theta}_{y}, \bar{\theta}_{z}\right]$ of the beam cross section by:

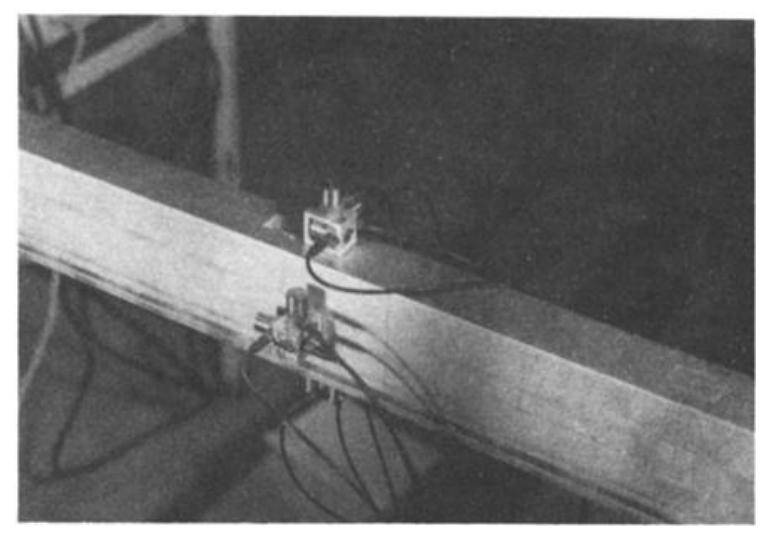

FIG. 7. Accelerometer array for beam modal analysis.

$$
\begin{gathered}
{\left[\begin{array}{c}
\bar{u}_{1} \\
\bar{v}_{1} \\
\bar{w}_{1} \\
\bar{u}_{2} \\
\bar{v}_{2} \\
\bar{w}_{2} \\
\bar{u}_{3} \\
\bar{v}_{3} \\
\bar{w}_{3}
\end{array}\right]=\left[\begin{array}{cccccc}
1 & 0 & 0 & 0 & z_{1} & -y_{1} \\
0 & 1 & 0 & -z_{1} & 0 & 0 \\
0 & 0 & 1 & y_{1} & 0 & 0 \\
1 & 0 & 0 & 0 & z_{2} & -y_{2} \\
0 & 1 & 0 & -z_{2} & 0 & 0 \\
0 & 0 & 1 & y_{2} & 0 & 0 \\
1 & 0 & 0 & 0 & z_{3} & -y_{3} \\
0 & 1 & 0 & -z_{3} & 0 & 0 \\
0 & 0 & 1 & y_{3} & 0 & 0
\end{array}\right]\left[\begin{array}{c}
\bar{u}_{0} \\
\bar{v}_{0} \\
\bar{w}_{0} \\
\bar{\theta}_{x} \\
\bar{\theta}_{y} \\
\bar{\theta}_{z}
\end{array}\right]} \\
=[A]\left[S_{0}\right],
\end{gathered}
$$

where $[A]$ is a $9 \times 6$ location matrix. For the selected accelerometer locations shown in Fig. 7, matrix $[A]^{T}[A]$ is nonsingular, and Eq. (5) has a unique solution. Therefore, $\left[S_{0}\right]$ can be calculated by

$$
\left[S_{0}\right]=\left([A]^{T}[A]\right)^{-1}[A]^{T}\left[\bar{u}_{1}, \bar{v}_{1}, \bar{w}_{1}, \bar{u}_{2}, \bar{v}_{2}, \bar{w}_{2}, \bar{u}_{3}, \bar{v}_{3}, \bar{w}_{3}\right]^{T} \text {. }
$$

\section{RESULTS AND DISCUSSION}

In the experiment, the beam was driven by the primary shaker at $970 \mathrm{~Hz}$. When the controller was implemented, the beam accelerations measured at the error sensor locations were minimized. Twenty five cross sections along the beam were selected to determine the global acceleration distribution for both controlled and uncontrolled conditions. As the beam was terminated anechoically, and the accelerometers to the right of the error sensor were in the far field of the control source, these acceleration measurements provided a measure of the power flow along the beam.

Figure 8(a) shows the three translation components $\left[\bar{u}_{0}, \bar{v}_{0}, \bar{w}_{0}\right]$ of the acceleration of the beam cross sections as a function of the beam length. By comparing the distributions of the acceleration level between the controlled and uncontrolled cases, it can be seen that effective control of all three translation components was achieved in the vicinity of the error sensors and to the right of the sensors, indicating that accelerometers mounted in a single plane provided an adequate error signal for minimization of power flow. However an increase of the acceleration level in the vicinity of the control actuators and the primary shaker can be seen. These increases in response in the vicinity of the control actuators also has been observed by others, ${ }^{3,4,13}$ and is due to the combined effects of the near field of the actuators, and the interference between the incident waves and reflected waves in the beam.

Figure $8(\mathrm{~b})$ shows the controlled and uncontrolled results for the three rotational motion components $\left[\bar{\theta}_{x}, \bar{\theta}_{y}, \bar{\theta}_{z}\right]$ distributed along the beam. Two components, $\bar{\theta}_{y}, \bar{\theta}_{z}$, which are related to the rotational parts of the two flexural waves in the $Y$ and $Z$ directions respectively, are controlled on the right-hand side of the error sensor. However, the component $\bar{\theta}_{x}$, which is related to torsional wave propagation along the beam, is not suppressed at all. This is expected, since the torsional wave has not been sensed in the error sensor sys- 

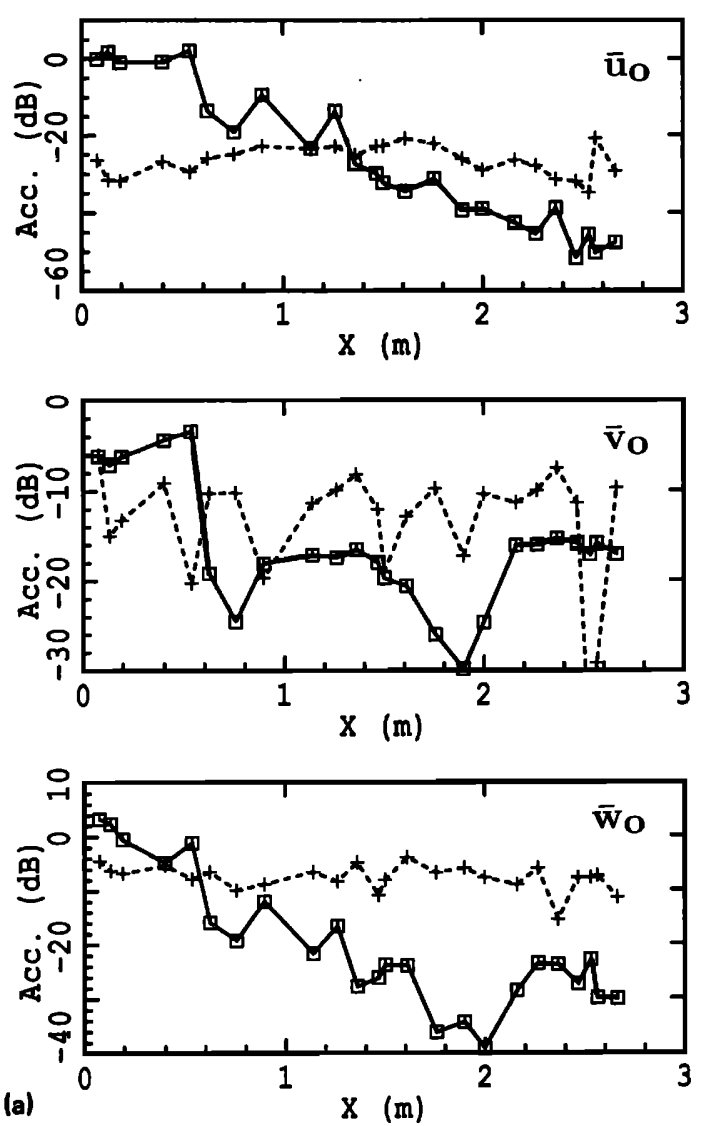

(a)
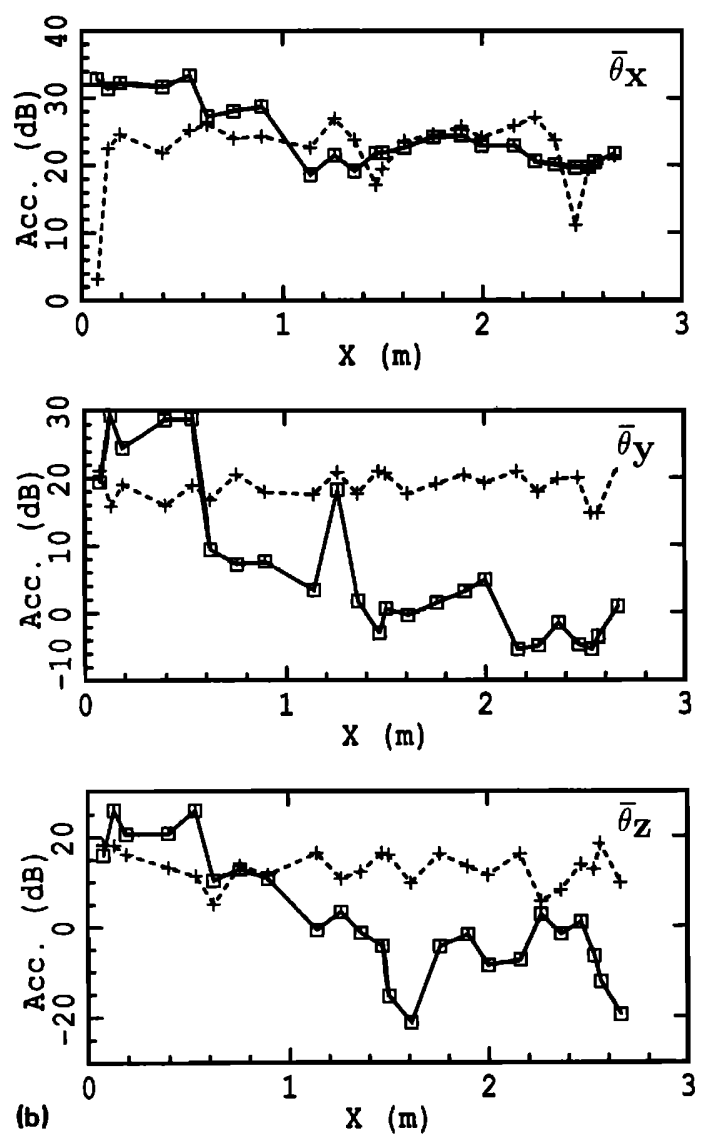

FIG. 8. Controlled and uncontrolled acceleration components on beam cross sections: (a) three translation components; (b) three rotational components. The excitation frequency was $970 \mathrm{~Hz}$. In all cases the dashed line represents the uncontrolled case, while the solid line represents the controlled case. tem, and the control actuator system has no effect upon this wave type. The experimental results suggest that the complete control of all the wave types requires a four-channel controller, and an extra set of error sensors and control actuators for the torsional waves.

In controlling each of the flexural waves in the beam, two components of each wave were suppressed with a single error sensor and actuator. (For example, for the $X-Y$ flexural wave, the two components were $\bar{v}_{0}, \bar{\theta}_{z}$ ). This is probably because the control actuator affected the flexural wave input impedance of the beam at the primary source, thus reducing the total flexural wave power flow input (which consists of rotational as well as translational motion components) even though the rotational motion was not observed at the error sensor.

\section{CONCLUSIONS}

Active control of two flexural waves and one extensional wave in a thick beam was achieved with a multichannel adaptive LMS algorithm implemented in a digital signal processor. The control actuators were piezoceramic patches bonded to the beam and driven so that flexural and extensional waves could be excited independently, while error information was obtained using accelerometers. It was found that to successfully control multiple wave types in a structure, error sensors and actuators capable of observing and controlling independent wave types must be implemented.

An array of accelerometers was used to determine the three translational and three rotational components of motion in the beam at various cross sections along the length of the beam. Results indicate that it is possible to achieve 30-dB attenuation of the structural response and vibratory power flow of all wave types downstream of the control actuators and disturbance. With the exception of rotation about the longitudinal axis of the beam, which was unaffected by the control actuators used in the experiments described here, all other translational and rotational responses were attenuated.

\section{ACKNOWLEDGMENTS}

Financial support for this work from the Australian Defence Science and Technology Organization, Material Research Laboratories is gratefully acknowledged. In addition the authors acknowledge the support of the Office of Naval Research and NASA Langley.

'B. R. Mace, "Active control of flexural vibration," J. Sound Vib. 114, 253 270 (1987).

${ }^{2}$ W. Redman-White, P. A. Nelson, and A. R. D. Curtis, "Experiments on the active control of flexural wave power flow," J. Sound Vib. 121, 187191 (1987).

${ }^{3}$ L. O. Gonidou, "Active control of flexural power flow in elastic thin beams," MS thesis, Virginia Polytechnic Institute and State University (1988).

${ }^{4}$ C. R. Fuller and L. O. Gonidou, "Active vibration control of flexural power flow in beams," J. Acoust. Soc. Am. Suppl. 1 84, S47 (1988).

${ }^{5}$ A. H. von Flotow and B. Schafer, "Wave-absorbing controller for a flexible system" J. Guidance, Control, Dynamics 9, 673-680 (1986). 
${ }^{6} \mathrm{~J}$. Pan and C. H. Hansen, "Active control of total power flow along a beam. I: Physical system analysis," J. Acoust. Soc. Am. 89, 200-209 (1990).

${ }^{7}$ C. H. Hansen and J. Pan, "Active control of beam vibration," in Proceedings of The Australian Vibration and Noise Conference, Melbourne, Sept. 1990 (Institution of Engineers (Australia), Barton, 1990).

${ }^{8}$ C. R. Fuller, G. P. Gibbs, and R. J. Silcox, "Simultaneous active control of flexural and extensional power flow in beams," Proceedings of the International Congress on Recent Developments in Air and Structure Borne Sound and Vibration, Auburn Univ., March 1990 (Technomic, Lancaster, 1990), pp. 657-662.

${ }^{9}$ E. F. Crawley and I. de Luis, "Use of piezoelectric actuators as elements of intelligent structures," AIAA J. 25(10), 1373-1385 (1987).

${ }^{10}$ S. J. Elliott and P. A. Nelson, "Algorithm for multichannel LMS adaptive filtering," Electron. Lett. 21, 979-981 (1985).

"S. J. Elliott, I. M. Stothers, and P. A. Nelson, "A multiple error algorithm and its application to the active control of sound and vibration," IEEE Trans. Acoust. Speech Signal Process. ASSP-35 (10), 1423-1434 (1987).

${ }^{12}$ J. Pan, C. H. Hansen, and R. L. Clark, Jr., "Experimental determination of the total vibratory power flow in an elastic beam," (in preparation).

${ }^{13} \mathrm{X}$. Pan and C. H. Hansen, "Effect of error sensor location and type on the active control of beam vibration," J. Sound Vib. 163 (2) (1993). 